\title{
Comparison of 6 cone-beam computed tomography systems for image quality and detection of simulated canine impaction-induced external root resorption in maxillary lateral incisors
}

\author{
Ali Alqerban, ${ }^{\text {a }}$ Reinhilde Jacobs, ${ }^{\mathrm{b}}$ Steffen Fieuws, ${ }^{\mathrm{c}}$ Olivia Nackaerts, ${ }^{\mathrm{d}}$ The SEDENTEXCT Project Consortium, \\ and Guy Willems ${ }^{f}$ \\ Leuven, Belgium
}

\begin{abstract}
Introduction: The most frequent adverse effect of canine impaction is resorption of the adjacent incisors. The subjective image quality and the radiographic diagnostic accuracy for detection of simulated canine-induced external root resorption lesions in maxillary lateral incisors were compared among 6 cone-beam computed tomography (CBCT) systems in vitro. Methods: A child cadaver skull in the early mixed dentition was obtained. This skull had an impacted maxillary left canine and allowed a reliable simulation. Simulated root resorption cavities were created in 8 extracted maxillary left lateral incisors by the sequential use of $0.16-\mathrm{mm}$ diameter round burs in the distopalatal root surface. Cavities of varying depths were drilled in the middle or apical thirds of each tooth root according to 3 setups: slight $(0.15,0.20$, and $0.30 \mathrm{~mm})$, moderate $(0.60$ and $1.00 \mathrm{~mm})$, and severe $(1.50,2.00$, and $3.00 \mathrm{~mm})$ resorption. The lateral incisors, including 2 intact teeth, were repositioned individually in the alveolus with approximal contacts to the impacted maxillary left canine. Six sets of radiographic images were obtained with 3D Accuitomo-XYZ Slice View Tomograph (J. Morita, Kyoto, Japan), Scanora 3D CBCT (Soredex, Tuusula, Finland), Galileos 3D Comfort (Sirona Dental Systems, Bensheim, Germany), Picasso Trio (E-WOO Technology, Giheung-gu, Republic of Korea), ProMax 3D (Planmeca OY, Helsinki, Finland), and Kodak 9000 3D (Trophy, Croissy-Beaubourg, France) for each tooth setup. The CBCT images were acquired and subsequently analyzed by 12 observers. Linear models for repeated measures were used to compare the CBCT systems for the image quality and the degree of agreement between the diagnosed severity of root resorption and the true severity. Results: The differences in the image quality between CBCT systems were statistically significant $(P<0.001)$. The root resorption scores between CBCT systems showed a significantly higher score for the ProMax when compared with the Galileos and the Kodak. However, the differences in agreement between the diagnosed severity of root resorption and the true severity for all resorption sizes were not significantly different $(P>0.05)$ among the different CBCT systems. Conclusions: High image quality is important when detecting root resorption. The CBCT systems used in this study had high accuracy with no significant differences between them in the detection of the severity of root resorption. (Am J Orthod Dentofacial Orthop 2011;140:e129-e139)
\end{abstract}

From Catholic University of Leuven, Leuven, Belgium.

${ }^{a}$ Resident, Department of Orthodontics, Faculty of Medicine, School of Dentistry, Oral Pathology and Maxillo-Facial Surgery.

brofessor and head of the Oral lmaging Center, Department of Periodontology, Faculty of Medicine, School of Dentistry, Oral Pathology and Maxillo-Facial Surgery. 'Statistical consultant, 1-BioStat, Department of Public Health.

${ }^{\mathrm{d} P}$ Postdoctoral researcher, Oral Imaging Center, Faculty of Medicine, School of Dentistry, Oral Pathology and Maxillo-Facial Surgery.

' Listing of partners on www.sedentexct.eu.

${ }^{f}$ Professor and head, Department of Orthodontics and Forensic Odontology, Faculty of Medicine, School of Dentistry, Oral Pathology and Maxillo-Facial Surgery.

The authors report no commercial, proprietary, or financial interest in the products or companies described in this article.
The research leading to these results received funding from the European Atomic Energy Community's Seventh Framework Program FP7/2007-2011 under grant agreement no 212246 (SEDENTEXCT: Safety and Efficacy of a New and Emerging Dental X-ray Modality).

Reprint requests to: Guy Willems, Faculty of Medicine, School of Dentistry, Oral Pathology and MaxilloFacial Surgery, Department of Orthodontics, Katholieke Universiteit Leuven, Kapucijnenvoer 7, 3000 Leuven, Belgium; e-mail, guy. willems@med.kuleuven.be.

Submitted, December 2010; revised and accepted, March 2011. 0889-5406/\$36.00

Copyright (C) 2011 by the American Association of Orthodontists. doi:10.1016/j.ajodo.2011.03.021 
$\mathrm{M}$ axillary lateral incisor root resorption is the most common adverse effect associated with an impacted maxillary canine. This process is progressive and irreversible. The degree of resorption depends on the nature and strength of the pressure produced by the impacted canine; it often remains asymptomatic. ${ }^{1}$ Resorption defects can be challenging to correctly diagnose and difficult to accurately assess. ${ }^{2}$ Root resorption is mostly found close to the maxillary canine or the dental follicle. ${ }^{3}$ Root resorption usually starts mildly in a specific area. Yet in time, it can extend in all directions and invade the entire root, making the prognosis of the tooth poor. The exact diagnosis of root resorption entails an essential stage to determine the treatment plan. The selection of an effective therapeutic protocol for orthodontic patients is necessary to ensure correct treatment and better treatment outcomes. Radiographic investigation is an essential part of the early diagnostic process for root resorption because a clinical examination without radiographic information is insufficient for treatment decisions.

In the past, conventional 2-dimensional (2D) radiographic imaging was the first choice and most commonly used clinical method for obtaining a primary diagnostic radiograph for the detection of root resorption in routine patients. Several 2D techniques have been widely used for the differential diagnosis of root resorption. ${ }^{4-9}$ Previous studies have shown that root resorption less than 0.60 $\mathrm{mm}$ in diameter and $0.30 \mathrm{~mm}$ in depth cannot be detected with 2D radiography. ${ }^{4,8}$ In addition, buccal or lingual resorption is less detectable in 2D radiographs. ${ }^{10}$ The use of conventional 2D radiography has been found to be inaccurate for the detection of mild resorption..$^{11,12}$ Furthermore, 2D radiography is also inadequate for representing the resorption lesions and their dimensions, which depend on the severity of the root resorption. ${ }^{13} \mathrm{~A}$ comparative study found that digital radiography is more sensitive in detecting external root resorption than conventional radiography. ${ }^{8}$ In contrast, other studies have found that the performance of digital systems is equal to that of conventional systems. ${ }^{9,14}$ Digital subtraction radiography has been shown to be superior to conventional radiography for detection of simulated external root resorption by eliminating anatomic noise. ${ }^{6,15}$

There is a great need for a more accurate diagnostic method for root resorption. The use of computed tomography (CT) has been suggested in the assessment of root resorption. CT offers 3-dimensional (3D) imaging and increases the detection rate of incisor root resorption caused by impacted canines. ${ }^{3,12,16,17}$ However, CT has some limitations in the detection of mild resorptions in the apical third. ${ }^{18}$

Cone-beam CT (CBCT) is a promising alternative because it provides 3D imaging of dental structures with submillimeter resolution images of high diagnostic quality. CBCT also has a short scanning time, reduced radiation exposure, and low cost compared with conventional CT. ${ }^{19}$ The diagnostic tasks for which these CBCT systems were mostly used in orthodontics included impacted teeth, ${ }^{20,21}$ temporomandibular joints, root proximity and resorption, ${ }^{22,23}$ tooth movement, cephalometric analysis, cleft lip and palate, planning for miniscrews, and orthodontic treatment planning. ${ }^{24,25}$ CBCT has been found to be useful for diagnosing root resorption caused by ectopically erupting teeth. ${ }^{20,21}$ Recent studies have compared conventional radiography with CBCT for the detection of root resorption. It was shown that the detection of root resorption was higher when using $\mathrm{CBCT}$ than with conventional radiography, and it was suggested that CBCT imaging is a reliable tool for the localization and detection of root resorption. ${ }^{26-28}$ Although these systems have rapidly developed while improving their overall image quality, all CBCT systems mainly vary according to their field of volume (FOV) and the detector type, with either image intensifier tube and charge-coupled device or flat-panel detector. It has been reported that detector type influenced image quality such as IIT/CCD has more artifacts and produces more noise compared with flat-panel detector systems. ${ }^{29,30}$ Moreover, FOV was found to be correlated to spatial resolution and contrast. ${ }^{31,32}$ The radiation dose varies substantially between CBCT systems depending on FOV and parameters. ${ }^{29,32}$ The size of voxels plays a role in determining image resolution, quality, and scanning and reconstruction times of CBCT images. ${ }^{30}$ Previous studies have only compared the effect of voxel size in 1 or 2 CBCT systems. ${ }^{33}$ It was found that the 3 voxel sizes did not affect the diagnostic performance for detection of external root resorption. ${ }^{33}$ The best option is to work with dose optimization and full justification to apply a low-dose CBCT technique that offers reasonable to excellent diagnostic accuracy.

The diagnostic ability of different CBCT systems in detecting root resorption caused by an impacted canine has not been sufficiently studied.

The purpose of this in-vitro study was to compare subjective image quality and radiographic diagnostic accuracy for detection of simulated external root resorption lesions caused by an impacted canine with 6 CBCT systems. 


\section{MATERIAL AND METHODS}

A child cadaver skull in the early mixed dentition phase was used; this skull had an impacted left maxillary canine (Fig 1). CBCT radiographs of the dry skull were taken in specific in-vitro conditions as described by Alqerban et al. ${ }^{26}$ Briefly, 8 setups were examined, each with a different maxillary left lateral incisor placed into the extraction site of the maxillary left lateral incisor. Each of the 8 selected lateral incisors was specifically modified to simulate the resorption process according to slight $(0.15,0.20$, and $0.30 \mathrm{~mm})$, moderate $(0.60$ and $1.00 \mathrm{~mm})$, and severe $(1.50,2.00$, and $3.00 \mathrm{~mm})$ resorption. In total, 8 cavities ranging in depth from 0.15 to $3.00 \mathrm{~mm}$ were created on the distopalatal surface of the root in the middle or apical third. In addition, 2 setups with intact lateral incisors were used (control group). Each tooth was then consecutively repositioned in the alveolus of the pediatric skull. ${ }^{26}$

The samples were scanned according to the protocols recommended by the manufacturers. The CBCT systems were 3D Accuitomo-XYZ Slice View Tomograph (J. Morita, Kyoto, Japan), Scanora 3D CBCT (Soredex, Tuusula, Finland), Galileos 3D Comfort (Sirona Dental Systems, Bensheim, Germany), Picasso Trio (E-WOO Technology, Giheung-gu, Republic of Korea), ProMax 3D (Planmeca OY, Helsinki, Finland), and Kodak 9000 3D (Trophy, Croissy-Beaubourg, France). The machine specifications, scanning protocols, and the FOVs for each CBCT are shown in Table 1. After image acquisition, all scans were exported as digital imaging and communications in medicine (DICOM) files and saved to a portable hard disk for later reconstruction. Images were exported and viewed with OnDemand3D software (version 1, CyberMed, Seoul, South Korea) that provided slices in the axial, coronal, and sagittal planes and 3D models. All exposures were made by the same operator (A.A.).

Ten images from 5 CBCT systems (Accuitomo, Scanora, Galileos, Promax, and Kodak) were produced. These were the images of the 8 lateral incisors with simulated resorption cavities and 2 sound lateral incisors (control teeth). However, for the Picasso CBCT system, only 6 teeth were scanned: 4 lateral incisors with resorption cavities $(0.15,0.20,0.30$, and $0.60 \mathrm{~mm})$ and 2 sound lateral incisors. In total, 56 CBCT images were acquired and subsequently analyzed in 2 sessions. The first evaluation session was by 8 postgraduate orthodontic residents, 2 orthodontic instructors, and 2 dental radiologists. All images were viewed by each observer as screen shots. The slice that best showed root resorption in the axial, coronal, and sagittal views was used. The images for all CBCT systems were standardized based on identifying identical anatomic structures at the same location.

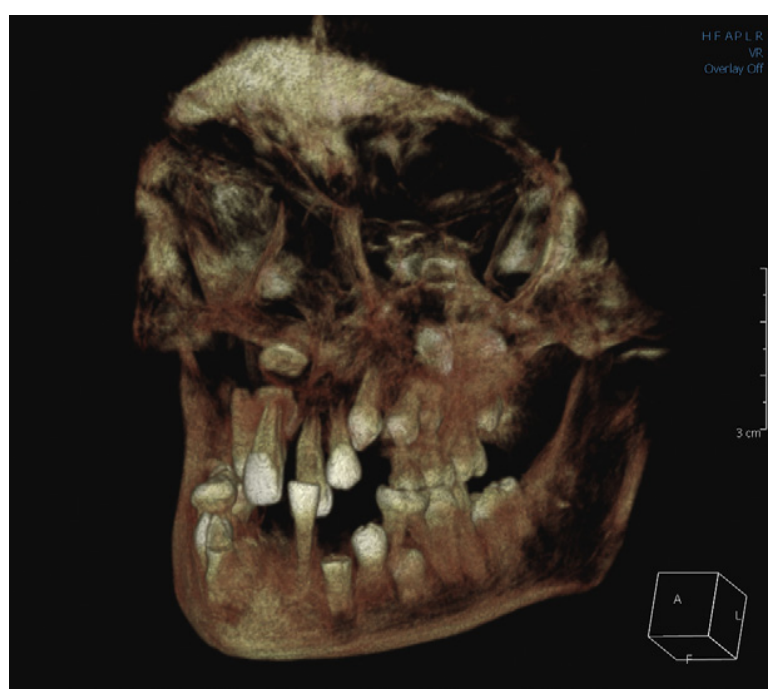

Fig 1. Three-dimensional image from Galileos 3D Comfort of the child cadaver skull in the early mixed dentition showing an impacted maxillary left canine in contact with the root surface of the maxillary lateral incisor.

To standardize viewing conditions, image brightness and contrast were calibrated by a light meter (PeakTech 5025, Dürr Dental, Bietigheim, Germany). The 12 observers were not allowed to adjust the brightness and contrast settings or the reconstruction views, thus ensuring standardized comparisons. All images were viewed on a 20-in flat panel screen $(2007$ FP $1600 \times 1200$, Brilliance 200WP, Philips, Brussels, Belgium). The acquired images in the first evaluation session were presented to the observers under the same conditions to prevent differences between observers while scoring the 6 CBCT systems. Observers used the screen shot and standardized contrast to decrease the role of other variables such as computer and viewing software experience and to become more user-friendly and efficient (Fig 2).

The second evaluation session was performed by the 2 dental radiologists, who reviewed the 56 DICOM data sets again 4 weeks after the first session. The radiologists in the second session were allowed to individually reconstruct the images with OnDemand3D and to adjust the brightness and contrast settings with software enhancement tools. They also were able to scroll through the CBCT slices using their own preferences for the optimal display of root resorption in the 3 planes (axial, coronal, and sagittal). This observation design was used to mimic the routine diagnostic approach in which clinicians can adjust image display settings.

All observers were blinded to the type of CBCT machine used and to the purpose of the study. The examiners were trained to use CBCT images for the detection 
Table I. Characteristics and technical specifications of the CBCT systems

$\begin{array}{lcccccc} & \text { Accuitomo } 3 D & \text { Scanora } & & & & \text { Kodak } \\ & \text { CBCT } & 3 D \text { CBCT } & \text { Galileos 3D } & \text { Picasso Trio } & \text { ProMax } 3 D & 90003 D \\ \text { Gray scale (bit) } & 8 & 12 & 12 & 12 & 12 & 14 \\ \text { Potential (kV) } & 80 & 85 & 85 & 85 & 84 & 85 \\ \text { Current (mA) } & 3 & 8-15 & 7 & 5 & 12 & 10 \\ \text { Exposure type } & \text { Continuous } & \text { Pulsed } & \text { Pulsed } & \text { Pulsed } & \text { Pulsed } & \text { Pulsed } \\ \text { Scan time (s) } & 18 & 2.25-4.5 & 3.4-14 & 15 & 18 & 10 \\ \text { Reconstruction time (min) } & 5 & 1-2 & 7 & 4-6 & 3 & 2 \\ \text { Voxel size (mm) } & 0.125 & 0.133-0.350 & 0.29 & 0.2 & 0.16 & 0.076-0.2 \\ \text { Field of volume (FOV) (mm) } & 30 \times 40 & 75 \times 100 & 120 \times 150 & 70 \times 120 & 80 \times 80 & 3.7 \times 50 \\ \text { Detector type } & \text { Image intensifier } & \text { Flat panel } & \text { Image intensifier } & \text { Flat panel } & \text { Flat panel } & \text { Flat panel }\end{array}$

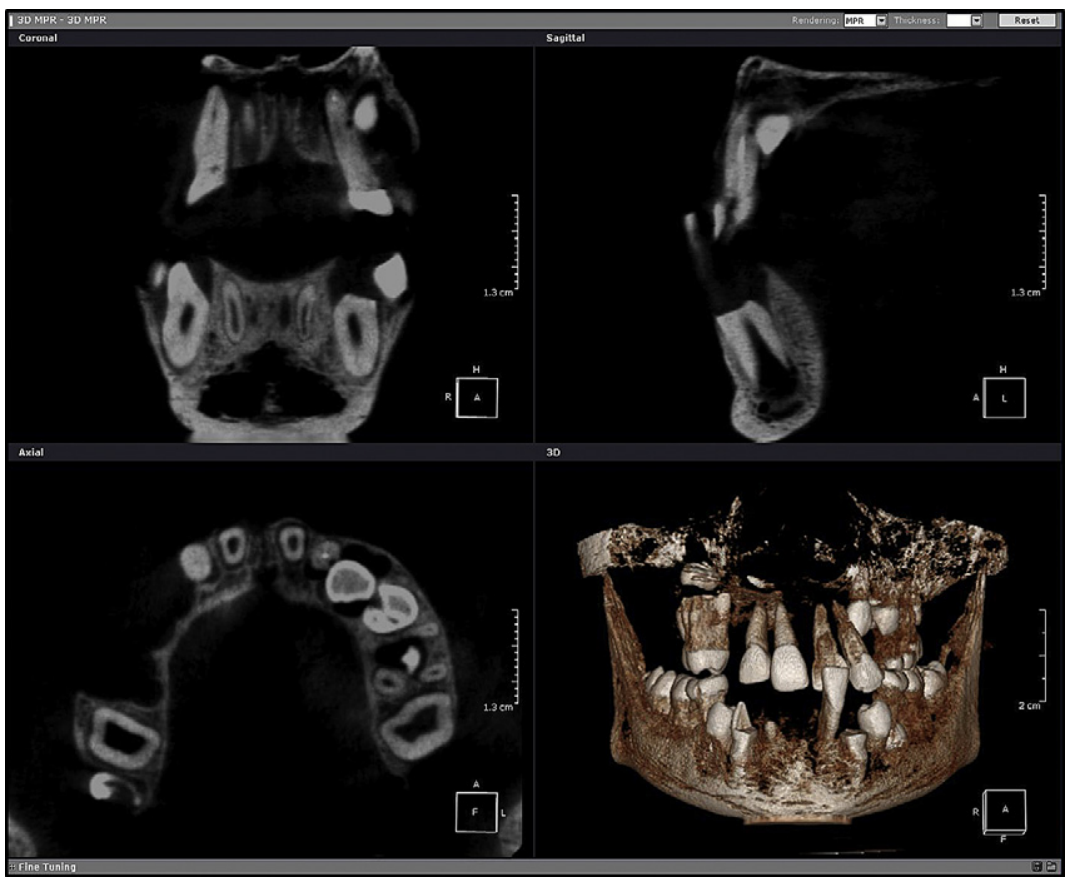

Fig 2. CBCT image (screen shot) obtained with Scanora 3D showing of axial, sagittal, coronal slices and the 3D model that were used in the first evaluation session to identify the 0.300 -mm resorption defect in the maxillary left lateral incisor.

of root resorption, and they assessed the images independently in the same random order. The observers were aware that not all images had root resorption, and they were encouraged to score only resorption in the root of the lateral incisor close to the impacted canine. The observation time was also not limited. The evaluation process of the 2 sessions included a questionnaire on the subjective diagnostic image quality on a 5-point rating scale (very poor, poor, acceptable, good, and excellent). This scale was used to assess the visibility of the following structures: pulp, dentin, and enamel; lamina dura; and overall image noise (brightness, contrast, and intensity). Excellent images were scored as those with clear visibility and distinguishable structures rather than esthetics of the image or the background.

After assessing the image quality, the observers were asked to assess the presence of a resorptive defect in the lateral incisor. The identification of root resorption was done by using a 5-step confidence scale: 1, definitely no resorption; 2 , maybe no resorption; 3 , unsure; 4, maybe resorption; 5, definitely resorption. If resorption was diagnosed, the examiners were asked to score the degree of resorption according to the criteria of Ericson and $\mathrm{Kurol}^{2}$ : slight (resorption up to half of the dentin thickness toward the pulp), moderate (resorption of half of the dentin thickness or more, with an unbroken pulp lining), or severe (pulp is exposed 
because of resorption). The examiners were then asked to classify the location of the diagnosed resorptive defect as apical, midapical (halfway between the apical and middle thirds), or middle third of the root.

\section{Statistical analysis}

In the first evaluation session, linear regression models for repeated measurements were used to analyze the relationships between the types of CBCT systems and various indexes of image quality (pulp, enamel, and dentin; lamina dura; overall image noise). Ordinal scores were treated as continuous variables in these analyses. An overall index of image quality was constructed by summing the scores of the 4 indexes. Tukey adjustments for multiple testing were used for pairwise comparisons between CBCT systems in the evaluation of image quality.

The observers' scores for root resorption were categorized so that scores of definitely resorption or maybe resorption were considered as "yes," and scores of unsure, maybe no resorption, or definitely no resorption were considered to be "no." With the actual presence of resorption used as the gold standard, the percentages of correct identification of resorption (sensitivity) and the percentages of correct identification of lack of resorption (specificity) were calculated. Sensitivity and specificity were compared among systems by using the McNemar test. A correction for multiple testing (false discovery rate) was applied on the set of pairwise comparisons between CBCT systems.

The linear regression model was used to compare the 8 setups with resorption for root resorption and to compare all 10 setups for agreement between the diagnosed severity of the resorption and the true severity. The agreement scores were calculated based on Table 1l. Perfect agreement was given a score of 3 , and most severe disagreement was given a score of 0 . Moreover, the linear regression model was also used for lesions of $0.6 \mathrm{~mm}$ or less to obtain a fair comparison with the Picasso CBCT systems.

All analyses were performed with SAS software (version 9.2, SAS System for Windows, SAS Institute, Cary, NC).

\section{RESULTS}

The image quality scores of pulp, dentin, and enamel; lamina dura; and overall image noise scored by $12 \mathrm{ob}-$ servers (first evaluation session) of each CBCT system are shown in Figure 3, $A$. The results of the second evaluation session of the image quality of dental structures by the 2 radiologists are shown in Figure 3, B. Figure 4 shows the sum of the image quality scores for each CBCT system. The differences in the image quality
Table II. Scores quantifying agreement between the diagnosed severity of root resorption and the true severity

\begin{tabular}{lcccc} 
& \multicolumn{4}{c}{$\begin{array}{c}\text { Score for severity of diagnosed lateral incisor } \\
\text { resorption }\end{array}$} \\
\cline { 2 - 5 } & $\begin{array}{c}\text { No } \\
\text { Resorption }\end{array}$ & $\begin{array}{c}\text { Slight } \\
\text { resorption }\end{array}$ & $\begin{array}{c}\text { Moderate } \\
\text { resorption }\end{array}$ & $\begin{array}{c}\text { Severe } \\
\text { resorption }\end{array}$ \\
True status & & & & \\
None & 3 & 2 & 1 & 0 \\
Slight & 2 & 3 & 2 & 1 \\
Moderate & 1 & 2 & 3 & 2 \\
Severe & 0 & 1 & 2 & 3 \\
\hline
\end{tabular}

scores between CBCT systems were statistically significant $(P<0.001)$ for all indexes including the sum of all quality scores (Fig 4, A). ProMax images had the best quality, with significantly higher scores than the other systems $(P<0.0001)$. The Galileos CBCT ranked as the second best system $(P<0.01)$. Moreover, the differences in scores between the CBCT systems followed a similar pattern in the second evaluation by the 2 radiologists (Fig $4, B$ ).

The radiologists rated image quality of the different CBCT systems higher than did the orthodontic observers (Table 111). The second evaluation session of the radiologists was better than the first evaluation session, thus indicating that freely using the software improved their ratings of the image quality (Table IIl, Fig 5).

The sensitivity and specificity for the root resorption results of the first and second evaluation sessions are reported in Tables IV and V. In the first evaluation session, the highest sensitivity was observed in the ProMax system; it was significantly higher than the sensitivity of the Galileos and Kodak systems $(P<0.01)$. Moreover, a significant difference $(P=0.003)$ was found between Scanora and Kodak. The overall specificity of detecting root resorption (percentage of control images scored as no resorption) was not significantly different among the CBCT systems $(P>0.05)$. It has been found that the lowest specificity was observed for the CBCT with the highest image quality (ProMax and Galileos). In the second evaluation session, sensitivity and specificity were not significantly different between the CBCT systems.

For the root resorption scores, significant differences were found in the first evaluation session between CBCT systems $(P=0.0004)$, with a higher score for ProMax compared to Galileos $(P=0.0005)$ and Kodak $(P=$ 0.005) (Fig 6, A). In the second evaluation session, no significant difference was found between the CBCT systems (Fig 6, B). 

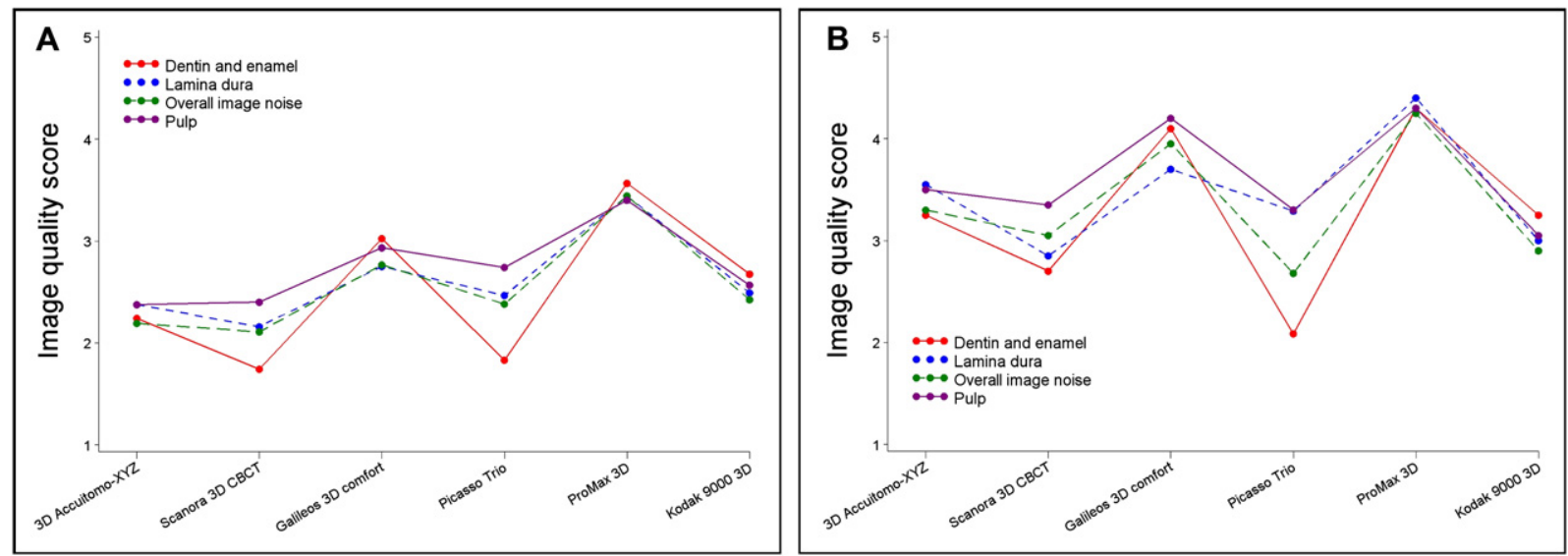

Fig 3. A, Image quality of dental structures scored by 12 observers based on images from 6 CBCT systems (first evaluation session); B, image quality of dental structures scored by 2 radiologist based on images from 6 CBCT systems (second evaluation session).
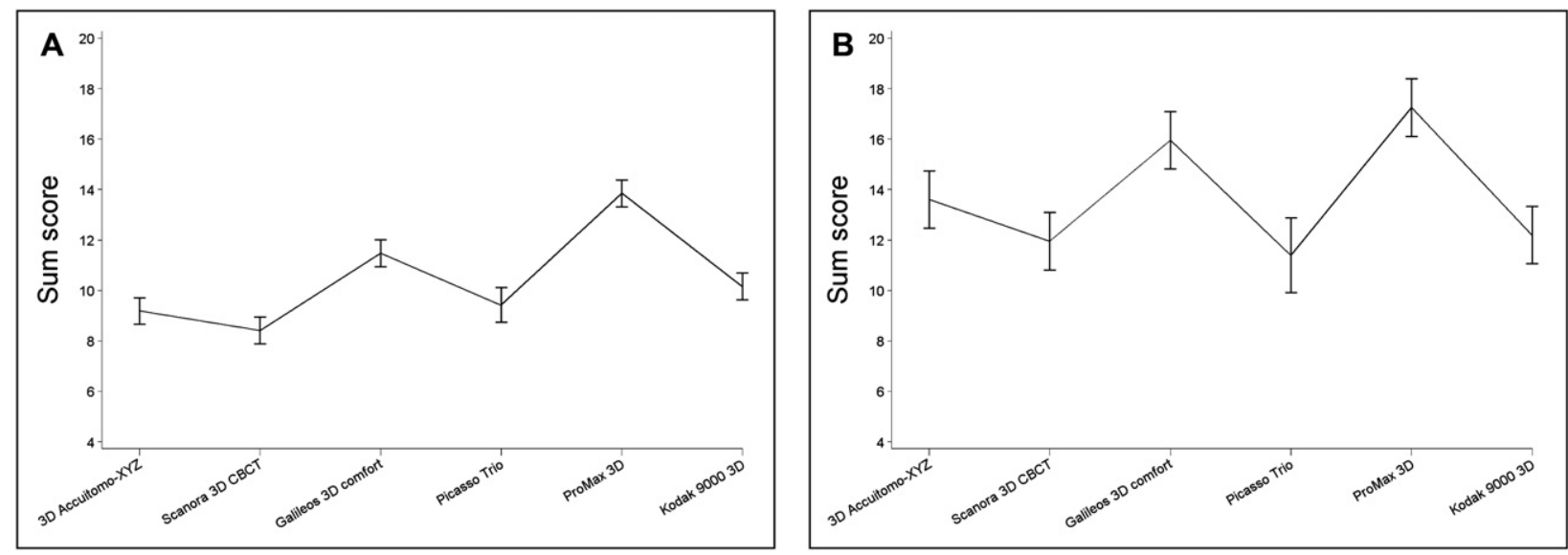

Fig 4. A, The sum of all image quality scores for anatomic structures of 6 CBCT systems scored by 12 observers (first evaluation session); $\mathbf{B}$, the sum of all image quality scores for anatomic structures of 6 CBCT systems scored by 2 radiologists (second evaluation session). Vertical lines denote the $95 \% \mathrm{Cl}$ values.

The degrees of root resorption, categorized as none, slight, moderate, and severe for the lateral incisor, with the 6 image systems are shown in Table Vl. For the ProMax CBCT imaging, $72.17 \%$ of the lateral incisor root cavities $(0.15,0.20$, and $0.30 \mathrm{~mm})$ were correctly classified as slight resorption. ProMax CBCT had the highest score for slight resorption, followed by Accuitomo and Galileos.

The distribution of the scores quantifying the agreement between the diagnosed severity of the resorption and the true severity for each CBCT system is shown in Table VIl. The differences in the agreement were not significantly different $(P>0.05)$ among the different CBCT systems (Fig 7).
In addition, the results of the presence and the agreement between the diagnosed severity of the root resorption had a similar pattern after the exclusion of images with lesions less than $0.6 \mathrm{~mm}$ among the CBCT systems.

\section{DISCUSSION}

Image quality has been extensively discussed in the literature. The assessment of root resorption caused by an impacted canine to the adjacent teeth by using images of high quality is essential to allow for the best visualization of early resorption and to decrease misinterpretation caused by image noise. The 3D imaging has been readily available for accurate, easily interpreted representations of root resorption. In previous 
Table III. Distribution (\%) of image quality scores from the observers

\begin{tabular}{|c|c|c|c|c|c|}
\hline & \multicolumn{4}{|c|}{ First evaluation } & \multirow{2}{*}{$\begin{array}{c}\text { Second evaluation } \\
\text { Radiologists } \\
(n=2)\end{array}$} \\
\hline & Scale & $\begin{array}{l}\text { Orthodontic residents } \\
\qquad(n=8)\end{array}$ & $\begin{array}{l}\text { Orthodontic instructors } \\
\qquad(n=2)\end{array}$ & $\begin{array}{l}\text { Radiologists } \\
\quad(n=2)\end{array}$ & \\
\hline \multirow[t]{5}{*}{ Pulp } & Very poor & 13.17 & 3.57 & 0.89 & 0.00 \\
\hline & Poor & 33.48 & 29.46 & 17.86 & 7.14 \\
\hline & Acceptable & 37.50 & 40.18 & 47.32 & 34.82 \\
\hline & Good & 14.29 & 25.89 & 27.68 & 44.64 \\
\hline & Excellent & 1.56 & 0.89 & 6.25 & 13.39 \\
\hline \multirow[t]{5}{*}{ Enamel and dentin } & Very poor & 18.75 & 15.18 & 10.71 & 4.46 \\
\hline & Poor & 34.15 & 33.93 & 29.46 & 10.71 \\
\hline & Acceptable & 28.35 & 30.36 & 33.04 & 40.18 \\
\hline & Good & 16.74 & 18.75 & 17.86 & 33.04 \\
\hline & Excellent & 2.01 & 1.79 & 8.93 & 11.61 \\
\hline \multirow[t]{5}{*}{ Lamina dura } & Very poor & 12.50 & 12.50 & 0.89 & 0.89 \\
\hline & Poor & 38.17 & 37.50 & 34.82 & 16.07 \\
\hline & Acceptable & 36.16 & 22.32 & 31.25 & 32.14 \\
\hline & Good & 11.83 & 25.89 & 27.68 & 36.61 \\
\hline & Excellent & 1.34 & 1.79 & 5.36 & 14.29 \\
\hline \multirow{5}{*}{$\begin{array}{l}\text { Overall structure identification on } \\
\text { the image quality }\end{array}$} & Very poor & 12.28 & 8.93 & 8.04 & 2.68 \\
\hline & Poor & 38.62 & 37.50 & 38.39 & 15.18 \\
\hline & Acceptable & 37.95 & 26.79 & 32.14 & 29.46 \\
\hline & Good & 10.94 & 25.89 & 14.29 & 44.64 \\
\hline & Excellent & 0.22 & 0.89 & 7.14 & 8.04 \\
\hline
\end{tabular}
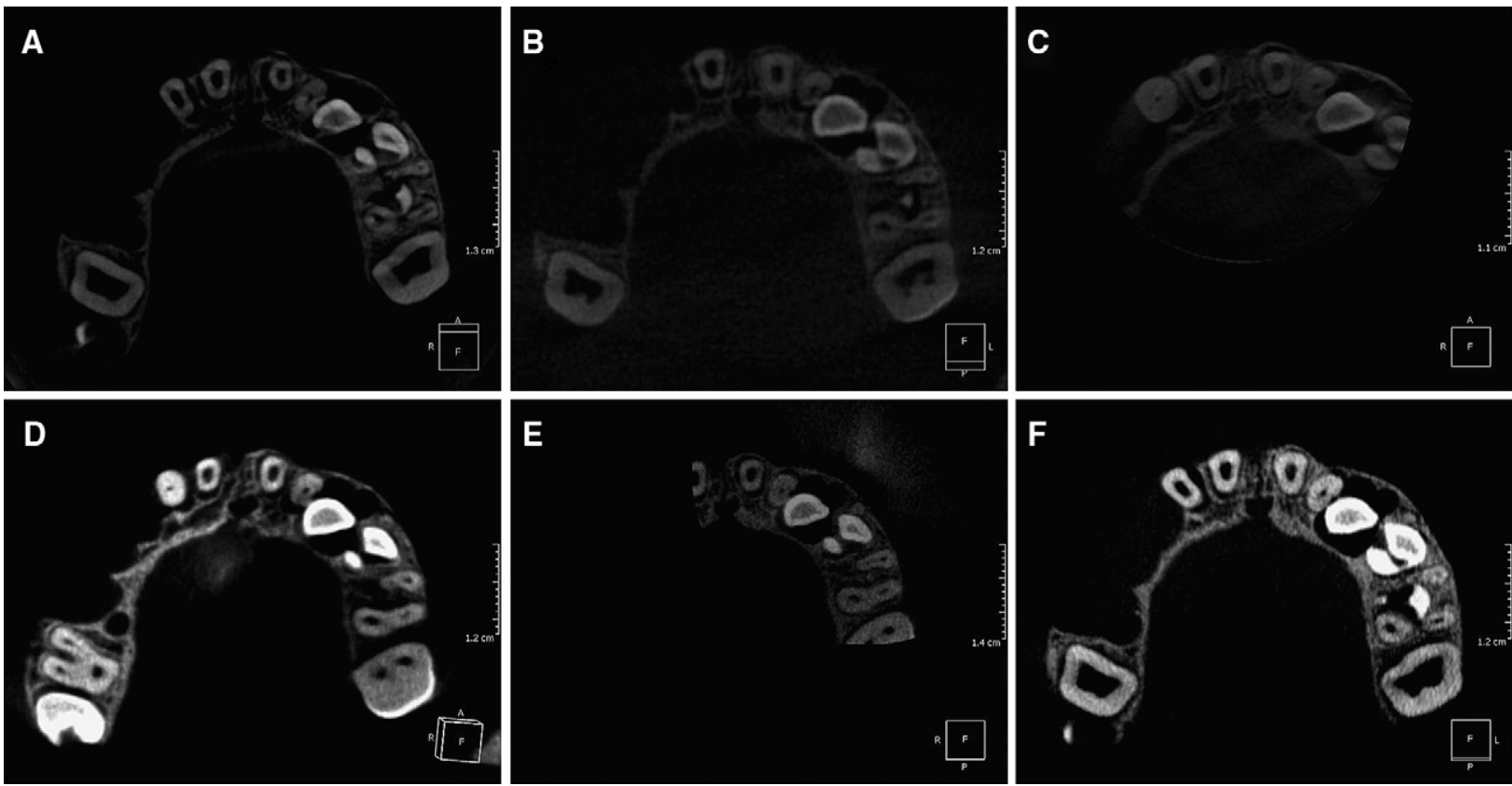

Fig 5. The axial slice sections showing the root resorption defect of $200 \mathrm{~mm}$ and image quality ranked by the 2 radiologists (A, ProMax 3D; B, Galileos 3D Comfort; C, 3D Accuitomo-XYZ; D, Scanora 3D СBCT; E, Kodak 9000 3D; F, Picasso Trio).

studies, CBCT has been used to evaluate the severity of resorptive lesions and found to be a reliable tool in diagnosis and treatment planning. ${ }^{27,34}$ Even with the advantages of CBCT over the conventional methods, the challenges of detecting root resorption are due to the difficulty of distinguishing between mild root 
Table IV. Sensitivity and specificity (\%) for the CBCT systems by 12 observers (first evaluation session), with sensitivity based on the 8 lateral incisors with simulated resorption cavities and specificity based on 2 sound lateral incisors

$\begin{array}{lcccccc}\text { CBCT system } & \text { Accuitomo } & \text { Galileos } & \text { Scanora } & \text { Kodak } & \text { Picasso } & \text { ProMax } \\ \text { Sensitivity } & 93.75 & 87.50 & 95.83 & 86.46 & 85.42 & 98.96 \\ \text { Specificity } & 87.50 & 70.83 & 95.83 & 91.67 & 95.83 & 58.33\end{array}$

Table V. Sensitivity and specificity (\%) for the CBCT systems by the 2 radiologists (second evaluation session), with sensitivity based on the 8 lateral incisors with simulated resorption cavities and specificity based on 2 sound lateral incisors

$\begin{array}{lcccccc}\text { CBCT system } & \text { Accuitomo } & \text { Galileos } & \text { Scanora } & \text { Kodak } & \text { Picasso } & \text { ProMax } \\ \text { Sensitivity } & 100 & 87.50 & 100 & 93.75 & 100 & 100 \\ \text { Specificity } & 75 & 100 & 50 & 100 & 100 & 75\end{array}$
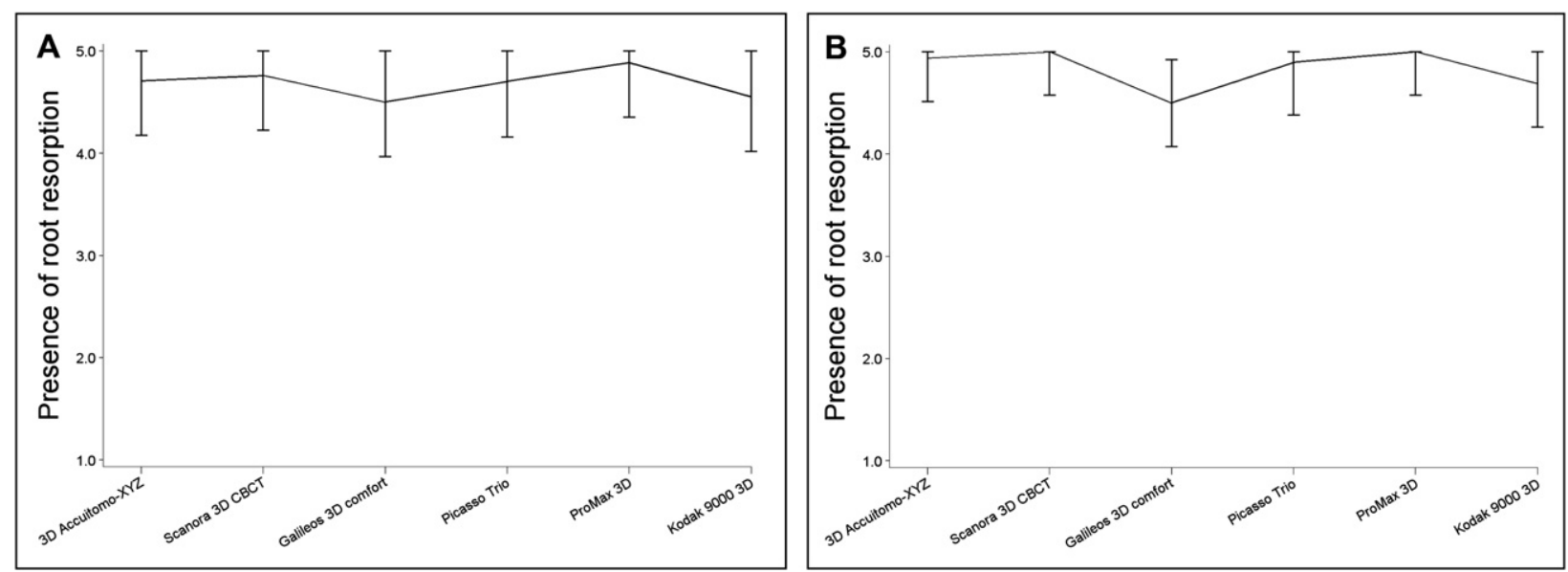

Fig 6. The difference between the 6 CBCT systems for root resorption: A, first evaluation session; $\mathbf{B}$, second evaluation session. Vertical lines denote the $95 \% \mathrm{Cl}$ values.

Table VI. Percentages of lateral incisor resorption correctly classified by the 12 observers

Degree of lateral incisor root resorption (\%)

\begin{tabular}{|c|c|c|c|c|}
\hline CBCT system & $\begin{array}{l}\text { No resorption } \\
\text { None }\end{array}$ & $\begin{array}{c}\text { Slight resorption } \\
0.15,0.20,0.30 \mathrm{~mm}\end{array}$ & $\begin{array}{c}\text { Moderate resorption } \\
0.60,1.00 \mathrm{~mm}\end{array}$ & $\begin{array}{c}\text { Severe resorption } \\
1.5,2.00,3.00 \mathrm{~mm}\end{array}$ \\
\hline Accuitomo & 79.17 & 66.67 & 50.00 & 83.33 \\
\hline Galileos & 62.50 & 66.67 & 41.67 & 94.44 \\
\hline Scanora & 87.50 & 61.11 & 37.50 & 94.44 \\
\hline Kodak & 79.17 & 58.33 & 33.33 & 100 \\
\hline Picasso & 75.00 & 63.89 & 66.67 & 0 \\
\hline ProMax & 58.33 & 72.22 & 41.67 & 100 \\
\hline
\end{tabular}

resorption and image artifacts. Previous studies comparing the subjective image quality of CBCT systems with conventional CT showed that the diagnostic image quality of CBCT is similar to or even better than that of $\mathrm{CT}^{31,35}$
The diagnostic performance of CBCT might highly depend on the parameter settings as well as the machines used. It was difficult to standardize the parameters of these 6 CBCT systems because each has its own parameters and settings. The CBCT settings in this study 
Table VII. Distribution of quantifying the agreement scores between the diagnosed severity of the resorption and the true severity for the CBCT the systems

\begin{tabular}{lcccc} 
& \multicolumn{4}{c}{ Agreement between resorption and severity (\%) } \\
\cline { 2 - 5 } CBCT system & 0 & 1 & 2 & 3 \\
Accuitomo & 0.00 & 2.50 & 26.67 & 70.83 \\
Galileos & 0.83 & 1.67 & 28.33 & 69.17 \\
Scanora & 0.00 & 3.33 & 25.00 & 71.67 \\
Kodak & 0.00 & 1.67 & 28.33 & 70.00 \\
Picasso & 0.00 & 2.78 & 29.17 & 68.06 \\
ProMax & 0.00 & 1.67 & 26.67 & 71.67 \\
$P>0.05$ for the comparison of the distribution between the 6 CBCT systems. 3. Perfect agreement; 0 , severe or no disagreement.
\end{tabular}
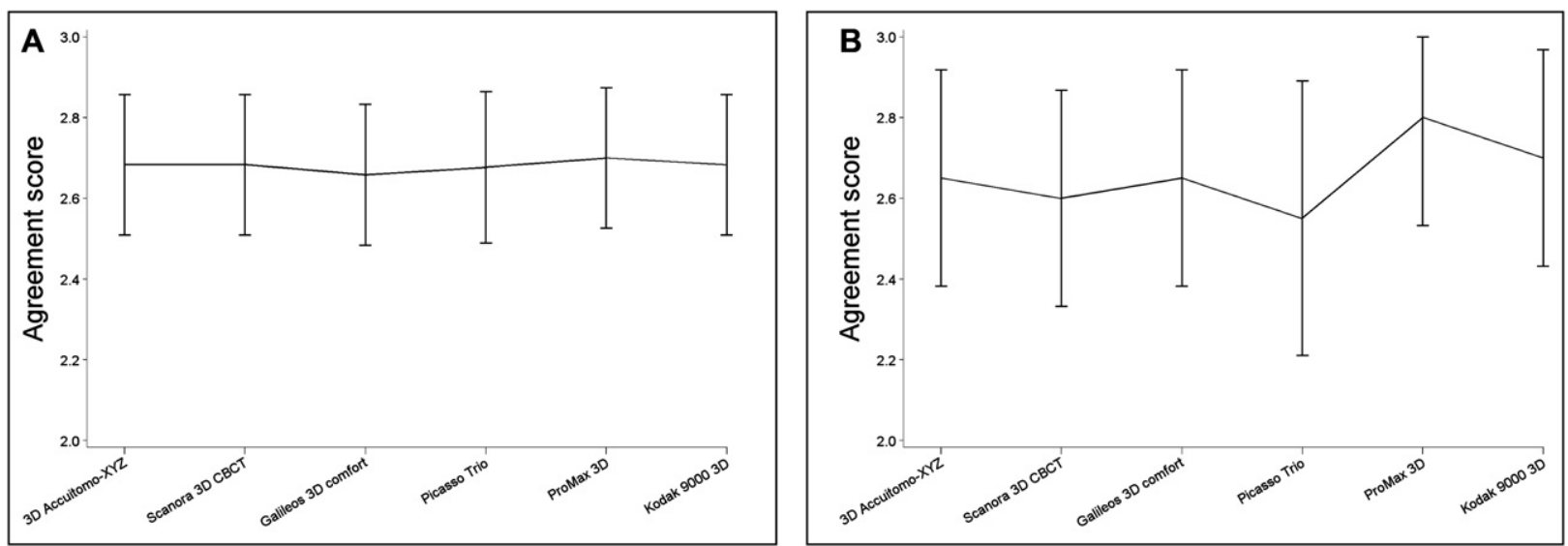

Fig 7. The difference between the 6 CBCT systems for agreement of the diagnosed severity of the resorption and the true severity: A, first evaluation session; B, second evaluation session. Vertical lines denote the $95 \% \mathrm{Cl}$ values.

were chosen according to each manufacturer's protocol. Although CBCT is expected to yield good results when detecting resorptions, its performance should be tested in various systems to validate how much information is gained for patients with impacted canines or root resorption. This study has established a link between image quality and the detection of simulated root resorption. Our null hypothesis was that 3D imaging with different CBCT systems is similar for detecting simulated root resorption.

The results of this study showed that the subjective image quality of the 6 CBCT systems was significantly different. CBCT systems were found to vary in image quality and visualization of anatomic structures. ${ }^{35,36}$ This agrees with our results. ProMax was the best system with regard to image quality, followed by Galileos. The 3D Accuitomo that was used in this study had an image intensifier with a charge-coupled device sensor having an 8-bit gray scale. A new model, 3D Accuitomo 80, was developed with a flat panel detector and a 13-bit gray scale that might give higher scores for contrast and image quality.
By using 5 scores for detection of root resorption (rather than yes or no), a better range might have been obtained. However, the overall results show high accuracy for root resorption detection in all CBCT systems. The overall sensitivity of CBCT systems was also high for detecting root resorption. The high sensitivity of CBCT systems is evidently the result of higher inherent contrast in CBCT images and the 3D view. However, there were significant differences in the determination of root resorption and sensitivity between ProMax and Galileos, and between ProMax and Kodak. The overall specificity was also high for all CBCT systems, except for the ProMax and Galileos, which had the highest image quality.

With respect to the agreement between the diagnosed severity of the resorption and the true severity, no significant difference was found among the CBCT systems tested. This means that there is no evidence for a difference among the systems regarding the precision of determining root resorption. The perfect agreement of the diagnosed severity of the resorption and the true severity of the cavities was high for all CBCT radiographic methods. The size and location of root 
resorption were reported to have a role in the accuracy of detection. ${ }^{12,18}$ However, in this study, these factors did not affect the accuracy of detection of root resorption. The differences in parameters and clinical usage of the CBCT systems tested were all relevant to orthodontics practice. The results show that all tested CBCT systems can be used to detect root resorption.

Scanning with Picasso CBCT was limited to lesions of $0.6 \mathrm{~mm}$ or less because of access and time limitations related to its use. Images of lesions greater than $0.6 \mathrm{~mm}$ $(1.00,1.50,2.00$, and $3.00 \mathrm{~mm})$ were considered as missing values. To evaluate whether including only 6 Picasso CBCT images would give a fair statistical comparison, a second analysis was based only on the images of the lesions of $0.6 \mathrm{~mm}$ or less. The results were similar for both analyses.

The radiologists evaluated the root resorption in 2 sessions: a screen-shot session and a software-and-scroll session. The performance of the radiologists in the second evaluation session was similar to that of all 12 observers. However, the radiologists' evaluations of image quality and detection of root resorption scored higher compared with the orthodontic instructors and the postgraduate residents because the radiologists had more experience and were more familiar with such images.

These results were similar to those of a previous invitro study that found no significant differences between CBCT systems in the detection of root resorption. ${ }^{26}$ Our findings demonstrate that the CBCT images tested in this study had high accuracy in the detection of root resorption. All CBCT systems used in this study had high accuracy. Artifacts in CBCT images might affect the diagnosis of root resorption; this is why high spatial resolution and minimal artifacts are important when trying to diagnose root resorption.

\section{CONCLUSIONS}

Based on the results of this study, the CBCT systems tested showed variable image qualities. This might have surely affected the detectability and diagnostic accuracy of root resorption lesions. All CBCT systems in this study showed high accuracy in the detection of root resorption. There was no significant difference among CBCT systems in the detection of the severity of root resorption.

\section{REFERENCES}

1. Fuss Z, Tsesis 1, Lin S. Root resorption-diagnosis, classification and treatment choices based on stimulation factors. Dent Traumatol 2003;19:175-82.

2. Ericson S, Kurol J. Incisor root resorptions due to ectopic maxillary canines imaged by computerized tomography: a comparative study in extracted teeth. Angle Orthod 2000;70:276-83.
3. Ericson S, Bjerklin K, Falahat B. Does the canine dental follicle cause resorption of permanent incisor roots? A computed tomographic study of erupting maxillary canines. Angle Orthod 2002; 72:95-104.

4. Andreasen FM, Sewerin 1, Mandel U, Andreasen J0. Radiographic assessment of simulated root resorption cavities. Endod Dent Traumatol 1987;3:21-7.

5. Goldberg F, De Silvio A, Dreyer C. Radiographic assessment of simulated external root resorption cavities in maxillary incisors. Endod Dent Traumatol 1998;14:133-6.

6. Hintze H, Wenzel A, Andreasen FM, Swerin 1. Digital subtraction radiography for assessment of simulated root resorption cavities. Performance of conventional and reverse contrast modes. Endod Dent Traumatol 1992;8:149-54.

7. Ericson S, Kurol J. Incisor resorption caused by maxillary cuspids. A radiographic study. Angle Orthod 1987;57:332-46.

8. Westphalen VP, Gomes de Moraes 1, Westphalen FH, Martins WD, Souza PH. Conventional and digital radiographic methods in the detection of simulated external root resorptions: a comparative study. Dentomaxillofac Radiol 2004;33:233-5.

9. Borg E, Kallqvist A, Grondahl K, Grondahl HG. Film and digital radiography for detection of simulated root resorption cavities. Oral Surg Oral Med Oral Pathol Oral Radiol Endod 1998;86: $110-4$.

10. Follin ME, Lindvall AM. Detection of lingual root resorptions in the intraoral radiographs. An experimental study. Swed Dent J 2005; 29:35-42.

11. Ericson S, Kurol PJ. Resorption of incisors after ectopic eruption of maxillary canines: a CT study. Angle Orthod 2000;70:415-23.

12. Preda L, La Fianza A, Di Maggio EM, Dore E, Schifino MR, Campani R, et al. The use of spiral computed tomography in the localization of impacted maxillary canines. Dentomaxillofac Radiol 1997;26:236-41.

13. Sameshima GT, Asgarifar KO. Assessment of root resorption and root shape: periapical vs panoramic films. Angle Orthod 2001; 71:185-9.

14. Kamburoglu K, Tsesis 1, Kfir A, Kaffe 1. Diagnosis of artificially induced external root resorption using conventional intraoral film radiography, CCD, and PSP: an ex vivo study. Oral Surg Oral Med Oral Pathol Oral Radiol Endod 2008;106:885-91.

15. Kravitz LH, Tyndall DA, Bagnell CP, Dove SB. Assessment of external root resorption using digital subtraction radiography. J Endod 1992;18:275-84.

16. Peene P, Lamoral Y, Plas H, Wilms G, De Bethune V, Opdebeeck H, et al. Resorption of the lateral maxillary incisor: assessment by CT. J Comput Assist Tomogr 1990;14:427-9.

17. Ericson S, Kurol J. Incisor root resorptions due to ectopic maxillary canines imaged by computerized tomography: a comparative study in extracted teeth. Angle Orthod 2000;70:276-83.

18. da Silveira HL, Silveira HE, Liedke GS, Lermen CA, Dos Santos RB, de Figueiredo JA. Diagnostic ability of computed tomography to evaluate external root resorption in vitro. Dentomaxillofac Radiol 2007;36:393-6.

19. Alqerban A, Jacobs R, Lambrechts P, Loozen G, Willems G. Root resorption of the maxillary lateral incisor caused by impacted canine: a literature review. Clin Oral Investig 2009;13:247-55.

20. Walker L, Enciso R, Mah J. Three-dimensional localization of maxillary canines with cone-beam computed tomography. Am J Orthod Dentofacial Orthop 2005;128:418-23.

21. Liu DG, Zhang WL, Zhang ZY, Wu YT, Ma XC. Localization of impacted maxillary canines and observation of adjacent incisor resorption with cone-beam computed tomography. Oral Surg Oral Med Oral Pathol Oral Radiol Endod 2008;105:91-8. 
22. Dudic A, Giannopoulou C, Leuzinger M, Kiliaridis S. Detection of apical root resorption after orthodontic treatment by using panoramic radiography and cone-beam computed tomography of super-high resolution. Am J Orthod Dentofacial Orthop 2009; 135:434-7.

23. Leuzinger M, Dudic A, Giannopoulou C, Kiliaridis S. Root-contact evaluation by panoramic radiography and cone-beam computed tomography of super-high resolution. Am J Orthod Dentofacial Orthop 2010;137:389-92.

24. Chen J, Li S, Fang S. Quantification of tooth displacement from cone-beam computed tomography images. Am J Orthod Dentofacial Orthop 2009;136:393-400.

25. Kau CH, Richmond S, Palomo JM, Hans MG. Three-dimensional cone beam computerized tomography in orthodontics. J Orthod 2005;32:282-93.

26. Alqerban A, Jacobs R, Souza PC, Willems G. In-vitro comparison of 2 cone-beam computed tomography systems and panoramic imaging for detecting simulated canine impaction-induced external root resorption in maxillary lateral incisors. Am J Orthod Dentofacial Orthop 2009;136:764.e1-11.

27. Patel S, Dawood A, Wilson R, Horner K, Mannocci F. The detection and management of root resorption lesions using intraoral radiography and cone beam computed tomography-an in vivo investigation. Int Endod J 2009;42:831-8.

28. Alqerban A, Jacobs R, Fieuws S, Willems G. Comparison of two cone beam computed tomographic systems versus panoramic imaging for localization of impacted maxillary canines and detection of root resorption. Eur J Orthod 2011;33:93-102.
29. Loubele M, Jacobs R, Maes F, Denis K, White S, Coudyzer W, et al. Image quality vs radiation dose of four cone beam computed tomography scanners. Dentomaxillofac Radiol 2008;37:309-18.

30. Scarfe WC, Farman AG, Sukovic P. Clinical applications of cone-beam computed tomography in dental practice. J Can Dent Assoc 2006;72:75-80.

31. Liang X, Jacobs R, Hassan B, Li L, Pauwels R, Corpas L, et al. A comparative evaluation of cone beam computed tomography (CBCT) and multi-slice CT (MSCT) Part 1. On subjective image quality. Eur J Radiol 2010;75:265-9.

32. Suomalainen A, Kiljunen T, Kaser Y, Peltola J, Kortesniemi M. Dosimetry and image quality of four dental cone beam computed tomography scanners compared with multislice computed tomography scanners. Dentomaxillofac Radiol 2009;38:367-78.

33. Liedke GS, da Silveira HE, da Silveira HL, Dutra V, de Figueiredo JA. Influence of voxel size in the diagnostic ability of cone beam tomography to evaluate simulated external root resorption. J Endod 2009;35:233-5.

34. Patel S, Dawood A. The use of cone beam computed tomography in the management of external cervical resorption lesions. Int Endod J 2007;40:730-7.

35. Loubele M, Guerrero ME, Jacobs R, Suetens P, van Steenberghe D. A comparison of jaw dimensional and quality assessments of bone characteristics with cone-beam CT, spiral tomography, and multi-slice spiral CT. Int J Oral Maxillofac Implants 2007;22:446-54.

36. Kwong JC, Palomo JM, Landers MA, Figueroa A, Hans MG. Image quality produced by different cone-beam computed tomography settings. Am J Orthod Dentofacial Orthop 2008;133:317-27. 